\title{
Manifest spacetime supersymmetry and the superstring
}

\author{
Nathan Berkovits \\ ICTP South American Institute for Fundamental Research, \\ Instituto de Fúsica Teórica, UNESP - Universidade Estadual Paulista, \\ Rua Dr. Bento T. Ferraz 271, 01140-070, São Paulo, SP, Brazil \\ E-mail: nathan.berkovits@unesp.br
}

ABSTRACT: The algebra of spacetime supersymmetry generators in the RNS formalism for the superstring closes only up to a picture-changing operation. After adding non-minimal variables and working in the "large" Hilbert space, the algebra closes without picturechanging and spacetime supersymmetry can be made manifest. The resulting non-minimal version of the RNS formalism is related by a field redefinition to the pure spinor formalism.

KEYwORDS: Superstrings and Heterotic Strings, Superspaces

ArXiv EPrint: 2106.04448 


\section{Contents}

1 Introduction 1

2 B-RNS-GSS formalism 2

3 Relation with pure spinor formalism 5

\section{Introduction}

Although the RNS formalism for the superstring has a beautiful geometric foundation coming from worldsheet super-reparameterization invariance, the lack of manifest spacetime supersymmetry complicates the computation of multiloop scattering amplitudes and the description of Ramond-Ramond backgrounds. As shown in [1], spacetime supersymmetry generators $q_{\alpha}$ can be constructed in this formalism which satisfy the algebra $\left\{q_{\alpha}, q_{\beta}\right\}=\gamma_{\alpha \beta}^{m} \tilde{P}_{m}$ where $\tilde{P}_{m}=\int d z e^{-\phi} \psi_{m}$.

The usual spacetime supersymmetry algebra would be $\left\{q_{\alpha}, q_{\beta}\right\}=\gamma_{\alpha \beta}^{m} P_{m}$ where $P_{m}=\int d z \partial x_{m}$ is the translation generator, and the operators $\tilde{P}_{m}$ and $P_{m}$ are related by an operation called "picture-changing". This operation changes the effective ground state for the bosonic worldsheet ghosts and can be used to change the "picture" of any physical operator, i.e. any operator which is BRST-invariant. But since the picture-changing operation which relates $\tilde{P}_{m}$ with $P_{m}$ is only valid on physical operators, the spacetime supersymmetry algebra only closes on onshell states which means that spacetime supersymmetry is not manifest.

On the other hand, the pure spinor formalism for the superstring [2] has manifest spacetime supersymmetry and can describe Ramond-Ramond backgrounds, but lacks a geometric foundation where the pure spinor BRST operator comes from gauge-fixing a worldsheet symmetry. There is complete agreement between all scattering amplitudes which have been computed using the two formalisms, but equivalence has not yet been proven and multiloop amplitudes in the two formalisms have different types of subtleties.

In this paper, the RNS and pure spinor formalisms will be shown to be equivalent by relating them to a manifestly spacetime supersymmetric generalization of the RNS formalism. In addition to the usual RNS matter and ghost variables, this generalization will involve non-minimal variables that include both pure spinor variables and the fermionic $\theta^{\alpha}$ and conjugate momenta $d_{\alpha}$ variables of the Green-Schwarz-Siegel formalism. ${ }^{1}$ Since the new formalism includes features of the pure spinor, RNS and Green-Schwarz-Siegel formalisms, it will be called the B-RNS-GSS formalism.

\footnotetext{
${ }^{1}$ The Green-Schwarz formalism is usually described with only $\left(x^{m}, \theta^{\alpha}\right)$ worldsheet variables, whereas the Siegel version of this formalism also includes the conjugate momenta $d_{\alpha}$ variables [3].
} 
The first step to making spacetime supersymmetry manifest in the RNS formalism is to work in the "large" Hilbert space including the ghost zero modes $\left(\xi_{0}, \eta_{0}\right)$ and to define the extended BRST operator $Q^{\prime}=Q_{\mathrm{RNS}}-\eta_{0}$. As discussed in [4] and [5], picture-changing can be interpreted as a BRST-trivial gauge transformation using $Q^{\prime}$ in the large Hilbert space. The next step is to add non-minimal variables to the RNS formalism and construct an operator $\rho_{\alpha}$ such that $\left\{q_{\alpha}^{\prime}, q_{\beta}^{\prime}\right\}=\gamma_{\alpha \beta}^{m} \int d z \partial x_{m}$ where $q_{\alpha}^{\prime}=q_{\alpha}^{\mathrm{RNS}}+Q^{\prime}\left(\rho_{\alpha}\right)$. And the final step is to find a similarity transformation which maps $q_{\alpha}^{\prime}$ into the spacetime supersymmetry generators of the Green-Schwarz-Siegel formalism.

The resulting worldsheet action and BRST operator for this B-RNS-GSS formalism are manifestly spacetime supersymmetric, and BRST-invariant vertex operators are easily constructed in terms of $d=10$ superfields. Furthermore, a field redefinition is found which maps this worldsheet action, BRST operator and vertex operators into the worldsheet action, BRST operator and vertex operators of the pure spinor formalism. It is hoped that by studying this intermediate B-RNS-GSS formalism, the relation between the multiloop subtleties in the RNS and pure spinor formalisms will be better understood.

\section{B-RNS-GSS formalism}

The B-RNS-GSS worldsheet action will be constructed from the usual RNS matter and ghost variables $\left(x^{m}, \psi^{m} ; b, c, \beta, \gamma\right)$ for $m=0$ to 9 , together with the Green-Schwarz-Siegel fermionic spacetime spinor variables $\left(\theta^{\alpha}, p_{\alpha}\right)$ for $\alpha=1$ to 16 , the unconstrained bosonic spacetime spinor variables $\left(\Lambda^{\alpha}, \Omega_{\alpha}\right)$, and the bosonic and fermionic pure spinor variables $\left(\bar{\lambda}_{\alpha}, \bar{w}^{\alpha}\right)$ and $\left(r_{\alpha}, s^{\alpha}\right)$ satisfying the pure spinor constraints $\bar{\lambda} \gamma^{m} \bar{\lambda}=\bar{\lambda} \gamma^{m} r=0$.

The left-moving contribution to the worldsheet action and stress tensor are

$$
\begin{aligned}
& S_{\text {B-RNS-GSS }}=S_{\text {RNS }}+\int d^{2} z\left[\Omega_{\alpha} \bar{\partial} \Lambda^{\alpha}+\bar{w}_{\alpha} \overline{\partial \lambda}^{\alpha}+p_{\alpha} \bar{\partial} \theta^{\alpha}+s^{\alpha} \bar{\partial} r_{\alpha}\right], \\
& T_{\text {B-RNS-GSS }}=T_{\text {RNS }}-\Omega_{\alpha} \partial \Lambda^{\alpha}-\bar{w}_{\alpha} \partial \bar{\lambda}^{\alpha}-p_{\alpha} \partial \theta^{\alpha}-s^{\alpha} \partial r_{\alpha},
\end{aligned}
$$

where the RNS worldsheet action, stress tensor, and BRST operator are defined as

$$
\begin{aligned}
S_{\mathrm{RNS}} & =\int d^{2} z\left(\frac{1}{2} \partial x^{m} \bar{\partial} x_{m}+\frac{1}{2} \psi^{m} \bar{\partial} \psi_{m}+\beta \bar{\partial} \gamma+b \bar{\partial} c\right), \\
T_{\mathrm{RNS}} & =-\frac{1}{2} \partial x^{m} \partial x_{m}-\frac{1}{2} \psi^{m} \partial \psi_{m}-\beta \partial \gamma-\frac{1}{2} \partial(\beta \gamma)-b \partial c-\partial(b c), \\
Q_{\mathrm{RNS}} & =\int d z\left(c T_{\mathrm{RNS}}+\gamma \psi^{m} \partial x_{m}+\gamma^{2} b-b c \partial c\right),
\end{aligned}
$$

and the right-moving variables will be ignored throughout this paper. Although only the open superstring will be discussed in this paper, all results can be easily generalized to the closed superstring by taking the "left-right product" of two open superstrings.

The natural BRST operator for this extended formalism is

$$
Q_{\mathrm{nonmin}}=Q_{\mathrm{RNS}}+\int d z\left[\Lambda^{\alpha} p_{\alpha}+\bar{w}^{\alpha} r_{\alpha}\right]
$$

since the non-minimal term $\int d z\left[\Lambda^{\alpha} p_{\alpha}+\bar{w}^{\alpha} r_{\alpha}\right]$ implies that states in the cohomology of $Q_{\text {nonmin }}$ are states in the cohomology of $Q_{\mathrm{RNS}}$ which are independent of the spacetime spinor variables $\left[\Lambda^{\alpha}, \Omega_{\alpha}, \theta^{\alpha}, p_{\alpha}, \bar{\lambda}_{\alpha}, \bar{w}^{\alpha}, r_{\alpha}, s^{\alpha}\right]$. 
In the RNS formalism [1], the spacetime supersymmetry generator in the $-\frac{1}{2}$ picture is $q_{\alpha}=\int d z e^{-\frac{1}{2} \phi} \Sigma_{\alpha}$ where the $(\beta, \gamma)$ ghosts have been fermionized as $\beta=\partial \xi e^{-\phi}$ and $\gamma=\eta e^{\phi}$, and $\Sigma_{\alpha}$ is the spin field of conformal weight $\frac{5}{8}$ constructed from $\psi^{m}$ by splitting the ten $\psi^{m}$ variables (after Wick rotation) into 5 complex variables $\psi_{ \pm}^{a}$ for $a=1$ to 5 , bosonizing $\psi_{ \pm}^{a}=e^{ \pm i \sigma_{a}}$, and defining $\Sigma_{\alpha}=e^{\frac{i}{2}\left( \pm \sigma_{1} \pm \sigma_{2} \pm \sigma_{3} \pm \sigma_{4} \pm \sigma_{5}\right)}$ with an even number of + signs. Since $\left\{q_{\alpha}, q_{\beta}\right\}=\gamma_{\alpha \beta}^{m} \int d z e^{-\phi} \psi_{m}$, the spacetime supersymmetry algebra only closes up to picture-changing. Note that $\left\{Q_{\mathrm{RNS}}, \int d z \xi e^{-\phi} \psi^{m}\right\}=\int d z \partial x^{m}$, so $\int d z e^{-\phi} \psi^{m}$ is the translation generator in the -1 picture where the picture $P$ is defined by $P=\int d z(\partial \phi-\xi \eta)$ so that $e^{n \phi}$ carries picture $P=n$ and the $(\beta, \gamma)$ ghosts carry picture $P=0$.

As discussed in [4] and [5], picture-changing can be treated as a BRST-trivial operation if one extends the space of states to the "large" Hilbert space which includes the zero mode of $\xi$ and defines the BRST operator as

$$
Q^{\prime}=Q_{\mathrm{RNS}}-\eta_{0}
$$

where $\eta_{0}=\int d z \eta$. Picture-changing from $V$ to $\left\{Q_{\mathrm{RNS}}, \xi V\right\}$ is expressed by the BRST-trivial operation $V \rightarrow V+Q^{\prime}(\xi V)=Q_{\mathrm{RNS}}(\xi V)$. And it is easy to show that any state in the cohomology of $Q^{\prime}$ can be expressed as a state in the small Hilbert space in the cohomology of $Q_{\mathrm{RNS}}$. For example, suppose that $Q^{\prime} V=0$ where $V=V_{P}+V_{P+1}$ and $V_{P}$ carries picture $P$. Then by separating the terms of different picture in $Q^{\prime} V=\left(Q_{\mathrm{RNS}}-\eta_{0}\right)\left(V_{P}+V_{P+1}\right)=0$, one finds

$$
\eta_{0} V_{P}=Q_{\mathrm{RNS}} V_{P}-\eta_{0} V_{P+1}=Q_{\mathrm{RNS}} V_{P+1}=0 .
$$

So $V^{\prime}=V+Q^{\prime}\left(\xi V_{P}\right)$ satisfies $Q_{\mathrm{RNS}} V^{\prime}=\eta_{0} V^{\prime}=0$.

By working in the large Hilbert space with $Q^{\prime}=Q_{\text {nonmin }}-\eta_{0}$ and shifting $q_{\alpha}$ by a BRST-trivial quantity, one can now make spacetime supersymmetry manifest by defining the generator

$$
q_{\alpha}=\int d z\left[e^{-\frac{1}{2} \phi} \Sigma_{\alpha}+Q^{\prime}\left(\rho_{\alpha}\right)\right]
$$

where $\rho_{\alpha}$ is chosen such that $\left\{q_{\alpha}, q_{\beta}\right\}=\int d z \partial x_{m} \gamma_{\alpha \beta}^{m}$ and will be discussed below. Furthermore, after performing a similarity transformation $q_{\alpha} \rightarrow e^{-R} q_{\alpha} e^{R}$, this generator can be mapped to the Green-Schwarz-Siegel supersymmetry generator [3]

$$
q_{\alpha}^{\mathrm{GSS}}=\int d z\left[p_{\alpha}+\frac{1}{2}\left(\partial x^{m}+\frac{1}{12} \theta \gamma^{m} \partial \theta\right)\left(\gamma_{m} \theta\right)_{\alpha}\right] .
$$

The resulting BRST operator $Q_{\mathrm{B}-\mathrm{RNS}-\mathrm{GSS}}=e^{-R}\left(Q_{\text {nonmin }}-\eta_{0}\right) e^{R}$ is manifestly spacetime supersymmetric and is

$$
\begin{aligned}
Q_{\text {B-RNS-GSS }}=\int d z[ & \Lambda^{\alpha} d_{\alpha}+\bar{w}^{\alpha} r_{\alpha}+\frac{1}{2} \xi e^{-\phi}\left(\Lambda \gamma^{m} \Lambda\right) \psi_{m}-e^{-\frac{1}{2} \phi} \Lambda^{\alpha} \Sigma_{\alpha} \\
& \left.+\gamma \psi^{m} \Pi_{m}+\gamma^{2}\left(b+\Omega_{\alpha} \partial \theta^{\alpha}+s^{\alpha} \partial \bar{\lambda}_{\alpha}\right)+c T-b c \partial c\right]-\eta_{0}
\end{aligned}
$$

where

$$
d_{\alpha}=p_{\alpha}-\frac{1}{2}\left(\partial x^{m}+\frac{1}{4} \theta \gamma^{m} \partial \theta\right)\left(\gamma_{m} \theta\right)_{\alpha}, \quad \Pi^{m}=\partial x^{m}+\frac{1}{2} \theta \gamma^{m} \partial \theta
$$


are the usual GSS spacetime supersymmetric operators [3] for fermionic and bosonic momenta. Note that $T$ of (2.2) can be written in the manifestly spacetime supersymmetric form $T=-\frac{1}{2} \Pi^{m} \Pi_{m}-d_{\alpha} \partial \theta^{\alpha}-\Omega_{\alpha} \partial \Lambda^{\alpha}-\bar{w}^{\alpha} \partial \bar{\lambda}_{\alpha}-s^{\alpha} \partial r_{\alpha}-\frac{1}{2} \psi^{m} \partial \psi_{m}-\beta \partial \gamma-\frac{1}{2} \partial(\beta \gamma)-b \partial c-\partial(b c)$.

To find the shift $\rho_{\alpha}$ and similarity transformation $R$ that map the supersymmetry generator and BRST operator into (2.10) and (2.11), first consider the shift and similarity transformation

$$
\begin{aligned}
\tilde{\rho}_{\alpha} & =-\Omega_{\alpha}+\frac{1}{2} \xi e^{-\phi} \psi^{m}\left(\gamma_{m} \theta\right)_{\alpha}, \\
\tilde{R} & =\int d z\left[c\left(\Omega_{\alpha} \partial \theta^{\alpha}+s^{\alpha} \partial \bar{\lambda}_{\alpha}\right)+e^{-\frac{1}{2} \phi} \Sigma_{\alpha} \theta^{\alpha}+\frac{1}{2} \xi e^{-\phi}\left(\theta \gamma^{m} \Lambda\right) \psi_{m}\right] .
\end{aligned}
$$

One finds that up to terms proportional to $\theta^{\alpha}, e^{-\tilde{R}} Q^{\prime} e^{\tilde{R}}$ is equal to $Q_{\text {B-RNS-GSS }}$ of $(2.11)$ and $e^{-\tilde{R}} \int d z\left[e^{-\frac{1}{2} \phi} \Sigma_{\alpha}+Q^{\prime}\left(\tilde{\rho}_{\alpha}\right)\right] e^{\tilde{R}}$ is equal to $q_{\alpha}^{\mathrm{GSS}}$ of $(2.10)$ where $Q^{\prime}=Q_{\text {nonmin }}-\eta_{0}$. But since $\theta^{\alpha}$ is a non-minimal variable, cohomology arguments imply that these terms proportional to $\theta^{\alpha}$ can be written as $\left[Q^{\prime}, f(\theta)\right]$ for some function $f(\theta)$ which is at least quadratic order in $\theta^{\alpha}$. For example, because of the term $\int d z \Lambda^{\alpha} p_{\alpha}$ in $Q^{\prime}$, any operator annihilated by $Q^{\prime}$ with linear dependence on $\theta^{\alpha}$ must be of the form $f_{\alpha \beta} \Lambda^{[\alpha} \theta^{\beta]}$ where $Q^{\prime} f_{\alpha \beta}=0$. But this is equal to $\left[Q^{\prime}, f_{\alpha \beta} \theta^{\alpha} \theta^{\beta}\right]$. Therefore, $Q_{\mathrm{B}-\mathrm{RNS}-\mathrm{GSS}}=e^{-R} Q^{\prime} e^{R}$ and $q_{\alpha}^{\mathrm{GSS}}=e^{-R} \int d z\left[e^{-\frac{1}{2} \phi} \Sigma_{\alpha}+Q^{\prime}\left(\rho_{\alpha}\right)\right] e^{R}$ where $R=\tilde{R}+f(\theta), \rho_{\alpha}=\tilde{\rho}_{\alpha}+f_{\alpha}(\theta)$, and $f$ and $f_{\alpha}$ are terms of quadratic or higher-order in $\theta^{\alpha}$.

Using the similarity transformation $R$, vertex operators in the cohomology of $Q_{\mathrm{B}-\mathrm{RNS}-\mathrm{GSS}}$ can be obtained from the usual RNS vertex operators as $V=e^{-R} V_{\mathrm{RNS}} e^{R}$. However, it is simpler to solve the condition that $Q_{\mathrm{B}-\mathrm{RNS}-\mathrm{GSS}} V=0$ and one finds that the unintegrated and integrated massless vertex operators are [6]

$$
\begin{aligned}
V= & \Lambda^{\alpha} A_{\alpha}(x, \theta)-\gamma \psi^{m} A_{m}(x, \theta)-\gamma^{2} \Omega_{\alpha} W^{\alpha}(x, \theta) \\
& +c\left[\partial \theta^{\alpha} A_{\alpha}(x, \theta)+\Pi^{m} A_{m}(x, \theta)+d_{\alpha} W^{\alpha}(x, \theta)\right. \\
& \left.-\frac{1}{2}\left(\psi^{m} \psi^{n}-\frac{1}{2} \Lambda \gamma^{m n} \Omega\right) F_{m n}(x, \theta)-\gamma \psi^{m} \Omega_{\alpha} \partial_{m} W^{\alpha}(x, \theta)\right], \\
\int d z U= & \int d z\left[\partial \theta^{\alpha} A_{\alpha}(x, \theta)+\Pi^{m} A_{m}(x, \theta)+d_{\alpha} W^{\alpha}(x, \theta)\right. \\
& \left.+\frac{1}{2}\left(-\psi^{m} \psi^{n}+\frac{1}{2} \Lambda \gamma^{m n} \Omega\right) F_{m n}(x, \theta)-\gamma \psi^{m} \Omega_{\alpha} \partial_{m} W^{\alpha}(x, \theta)\right],
\end{aligned}
$$

where $\left(A_{\alpha}, A_{m}, W^{\alpha}, F_{m n}\right)$ are the usual $d=10$ super-Yang-Mills superfields satisfying

$$
\begin{aligned}
D_{\alpha} A_{\beta}+D_{\beta} A_{\alpha} & =\gamma_{\alpha \beta}^{m} A_{m}, \quad D_{\alpha} A_{m}-\partial_{m} A_{\alpha}=\left(\gamma_{m}\right)_{\alpha \beta} W^{\beta}, \\
D_{\alpha} W^{\beta} & =\frac{1}{2}\left(\gamma^{m n}\right)_{\alpha}{ }^{\beta} \partial_{m} A_{n}=\frac{1}{4}\left(\gamma^{m n}\right)_{\alpha}{ }^{\beta} F_{m n},
\end{aligned}
$$

and $D_{\alpha}=\frac{\partial}{\partial \theta^{\alpha}}+\frac{1}{2}\left(\gamma^{m} \theta\right)_{\alpha} \partial_{m}$ is the $d=10$ supersymmetric derivative. 
Note that the similarity transformation of (2.15) mixes different pictures, but one can choose a representative of the BRST cohomology so that the massless super-Yang-Mills vertex operator $V$ of (2.16) is in the zero picture. And by constructing massive superstring vertex operators from the OPE's of massless vertex operators, one can similarly find vertex operators at zero picture for all GSO-projected onshell superstring states. For states which are not GSO-projected, i.e. which have square-root cuts with $e^{-\frac{1}{2} \phi} \Sigma_{\alpha}$, the vertex operator will need to involve spin fields in $\Lambda^{\alpha}$ and $\theta^{\alpha}$ so that the operator does not have square-root cuts with the term $\int d z \Lambda^{\alpha}\left(d_{\alpha}-e^{-\frac{1}{2} \phi} \Sigma_{\alpha}\right)$ in the BRST operator of (2.11).

For the gluon component of the superfield with polarization $\epsilon_{m}$, one finds from (2.16) that

$$
V=\epsilon_{m} e^{i k \cdot x}\left[V_{\mathrm{RNS}}^{m}-\frac{i}{2} c k_{n}\left(\Lambda \gamma^{m n} \Omega+\theta \gamma^{m n} p\right)+\mathcal{O}^{m}(\theta)\right]
$$

where $V_{\mathrm{RNS}}^{m}=-\gamma \psi^{m}+c\left(\partial x^{m}-i k_{n} \psi^{n} \psi^{m}\right)$ is the usual RNS vertex operator and $\mathcal{O}^{m}(\theta)$ denotes terms which are linear or higher-order in $\theta^{\alpha}$. It is easy to verify that the terms $\mathcal{O}^{m}(\theta)$ and $\left(\Lambda \gamma^{m n} \Omega+\theta \gamma^{m n} p\right)$ in (2.19) decouple from gluon scattering amplitudes since there are no extra $d_{\alpha}$ 's to contract with the $\mathcal{O}^{m}(\theta)$ term and since contractions of $\left(\Lambda \gamma^{m n} \Omega+\theta \gamma^{m n} p\right)$ with itself cancel because of opposite signs from the bosonic and fermionic contributions. So the scattering amplitudes for external gluons in the B-RNS-GSS formalism are identical to the amplitudes in the usual RNS formalism.

However, the vertex operator for the gluino component of the superfields in (2.16) is very different from the gluino vertex operator in the RNS formalism. Just as the spacetime supersymmetry generator of (2.10) does not involve the spin field $\Sigma_{\alpha}$, the gluino vertex operator in the B-RNS-GSS formalism does not involve the spin field. So the amplitude computation for external fermions looks very different from the RNS computation. Nevertheless, the two computations are guaranteed to give equivalent results since they are related by a similarity transformation.

\section{Relation with pure spinor formalism}

In this section, the BRST operator of (2.11) in the B-RNS-GSS formalism will be mapped to the pure spinor BRST operator by a field redefinition and similarity transformation. The field redefinition will "dynamically twist" the RNS variables $\psi^{m}$ of spin $\frac{1}{2}$ and $(\beta, \gamma)$ of spin $\left(\frac{3}{2},-\frac{1}{2}\right)$ into the variables $\left(\Gamma^{m}, \bar{\Gamma}_{m}\right)$ of spin $(0,1)$ and $(\widehat{\beta}, \widehat{\gamma})$ of spin $(2,-1)[7]$. The twisting of the $\psi^{m}$ variables to $\left(\Gamma^{m}, \bar{\Gamma}_{m}\right)$ variables shifts their central charge contribution from +5 to -10 , and is compensated by the replacement of the $(\beta, \gamma)$ with $(\widehat{\beta}, \widehat{\gamma})$ variables which shifts their central charge contribution from +11 to +26 . After performing a similarity transformation, the BRST operator of (2.11) in terms of these twisted variables is mapped to $Q_{\text {pure }}-\widehat{\eta}_{0}$ where $Q_{\text {pure }}=\int d z\left(\lambda^{\alpha} d_{\alpha}+\bar{w}^{\alpha} r_{\alpha}\right)$ is the pure spinor BRST operator and $\widehat{\eta}_{0}$ is the zero mode coming from fermionizing $\widehat{\beta}=\partial \widehat{\xi} e^{-\widehat{\phi}}$ and $\widehat{\gamma}=\widehat{\eta} e^{\widehat{\phi}}$.

To dynamically twist the ten $\psi^{m}$ spin-half variables into five spin-zero and five spin-one variables, it will be useful to construct a pure spinor $\lambda^{\alpha}$ out of $\Lambda^{\alpha}$ and $\bar{\lambda}_{\alpha}$ as

$$
\lambda^{\alpha}=\Lambda^{\alpha}-\frac{1}{2(\Lambda \bar{\lambda})}\left(\Lambda \gamma^{m} \Lambda\right)\left(\gamma_{m} \bar{\lambda}\right)^{\alpha}
$$


So $\lambda^{\alpha}$ and $\bar{\lambda}_{\alpha}$ satisfy $\lambda \gamma^{m} \lambda=\bar{\lambda} \gamma^{m} \bar{\lambda}=0$, and their 11 complex components (in Wick-rotated Euclidean space) parameterize the complex space $\frac{\mathrm{SO}(10)}{\mathrm{U}(5)} \times C$. Using the pure spinor variables $\left(\lambda^{\alpha}, \bar{\lambda}_{\alpha}\right)$ to covariantly choose the direction of the twisting, one can now dynamically twist the ten spin-half $\psi^{m}$ variables to spin-zero $\Gamma^{m}$ variables and spin-one $\bar{\Gamma}^{m}$ variables defined by

$$
\Gamma^{m}=\frac{1}{2(\lambda \bar{\lambda})} \gamma\left(\lambda \gamma^{m} \gamma^{n} \bar{\lambda}\right) \psi_{n}, \quad \bar{\Gamma}^{m}=\frac{1}{2(\lambda \bar{\lambda})} \frac{1}{\gamma}\left(\bar{\lambda} \gamma^{m} \gamma^{n} \lambda\right) \psi_{n}
$$

so that

$$
\psi^{m}=\gamma \bar{\Gamma}^{m}+\frac{1}{\gamma} \frac{\left(\lambda \gamma^{m} \gamma^{n} \bar{\lambda}\right)}{2(\lambda \bar{\lambda})} \Gamma_{n} .
$$

$\bar{\Gamma}^{m}$ will be constrained to satisfy $\bar{\Gamma}^{m}\left(\gamma_{m} \bar{\lambda}\right)^{\alpha}=0$, and since $\psi^{m}$ of (3.3) is invariant under the gauge transformation $\delta \Gamma_{m}=\epsilon \gamma_{m} \bar{\lambda}$ generated by this constraint, only half of the $\Gamma^{m}$ and $\bar{\Gamma}^{m}$ components are independent.

After expressing $\psi^{m}$ in terms of $\Gamma^{m}$ and $\bar{\Gamma}^{m}$, GSO-projected states only depend on even powers of the $\gamma$ ghost. So it will be useful to define

$$
\widehat{\gamma} \equiv(\gamma)^{2}
$$

which carries conformal weight -1 , and define $\widehat{\beta}$ of conformal weight +2 to be the conjugate momentum to $\widehat{\gamma}$. Fermionizing $(\widehat{\gamma}, \widehat{\beta})$ as $\widehat{\gamma}=\widehat{\eta} e^{\widehat{\phi}}$ and $\widehat{\beta}=\partial \widehat{\xi} e^{-\widehat{\phi}}$ and requiring that $(\widehat{\eta}, \widehat{\xi}, \widehat{\phi})$ have no poles with $\Gamma^{m}$ or $\bar{\Gamma}_{m}$, one finds that

$$
\begin{aligned}
\widehat{\eta} & =e^{-\frac{1}{2} \phi} \lambda^{\alpha} \Sigma_{\alpha}, & \widehat{\xi} & =e^{\frac{1}{2} \phi} \frac{1}{(\lambda \bar{\lambda})} \bar{\lambda}_{\alpha} \Sigma^{\alpha}, \\
e^{\widehat{\phi}} & =\eta \partial \eta e^{\frac{5}{2} \phi} \frac{1}{(\lambda \bar{\lambda})} \bar{\lambda}_{\alpha} \Sigma^{\alpha}, & e^{-\widehat{\phi}} & =\xi \partial \xi e^{-\frac{5}{2} \phi} \lambda^{\alpha} \Sigma_{\alpha} .
\end{aligned}
$$

One can also express the unhatted ghost variables in terms of the twisted variables as

$$
\eta=e^{-2 \widehat{\phi}} \frac{\left(\bar{\lambda} \gamma_{m n p q r} \bar{\lambda}\right)}{120(\lambda \bar{\lambda})^{2}} \Gamma^{m} \Gamma^{n} \Gamma^{p} \Gamma^{q} \Gamma^{r}, \quad \xi=\frac{1}{120} e^{2 \widehat{\phi}}\left(\lambda \gamma^{m n p q r} \lambda\right) \bar{\Gamma}_{m} \bar{\Gamma}_{n} \bar{\Gamma}_{p} \bar{\Gamma}_{q} \bar{\Gamma}_{r}
$$

In terms of these twisted variables, the BRST operator of (2.11) is

$$
\begin{aligned}
Q_{\text {B-RNS-GSS }}=\int d z[ & \lambda^{\alpha} d_{\alpha}+\bar{w}^{\alpha} r_{\alpha}+\Gamma^{m} \frac{\left(\bar{\lambda} \gamma_{m} \gamma_{n} \lambda\right)}{2(\lambda \bar{\lambda})} \Pi^{n}-\widehat{\eta}-e^{-2 \widehat{\phi}} \frac{\left(\bar{\lambda} \gamma_{m n p q r} \bar{\lambda}\right)}{120(\lambda \bar{\lambda})^{2}} \Gamma^{m} \Gamma^{n} \Gamma^{p} \Gamma^{q} \Gamma^{r} \\
& +u^{m}\left(\bar{\Gamma}_{m}+\frac{\left(\bar{\lambda} \gamma_{m} d\right)}{2(\lambda \bar{\lambda})}-\widehat{\xi} e^{-2 \widehat{\phi}} \frac{\left(\bar{\lambda} \gamma_{m n p q r} \bar{\lambda}\right)}{24(\lambda \bar{\lambda})^{2}} \Gamma^{n} \Gamma^{p} \Gamma^{q} \Gamma^{r}\right) \\
& \left.+\widehat{\gamma}\left(b+\bar{\Gamma}_{m} \Pi^{m}+\Omega_{\alpha} \partial \theta^{\alpha}+s^{\alpha} \partial \bar{\lambda}_{\alpha}\right)+c T-b c \partial c\right]
\end{aligned}
$$

where $u_{m}$ is defined by $\Lambda^{\alpha}=\lambda^{\alpha}+\frac{1}{2(\lambda \bar{\lambda})} u^{m}\left(\gamma_{m} \bar{\lambda}\right)^{\alpha}$,

$$
\begin{aligned}
T= & -\frac{1}{2} \partial x^{m} \partial x_{m}-\bar{\Gamma}_{m} \partial \Gamma^{m}-\widehat{\beta} \partial \widehat{\gamma}-\partial(\widehat{\beta} \widehat{\gamma})-b \partial c-\partial(b c) \\
& -p_{\alpha} \partial \theta^{\alpha}-v_{m} \partial u^{m}-w_{\alpha} \partial \lambda^{\alpha}-\widehat{\bar{w}}^{\alpha} \partial \bar{\lambda}_{\alpha}-s^{\alpha} \partial r_{\alpha}
\end{aligned}
$$


$\widehat{\beta}=\frac{1}{2 \gamma} \beta-\frac{1}{2 \gamma^{2}} \bar{\Gamma}_{m} \Gamma^{m}, v_{m}$ and $\bar{\Gamma}_{m}$ are constrained to satisfy $v_{m}\left(\gamma^{m} \bar{\lambda}\right)^{\alpha}=\bar{\Gamma}_{m}\left(\gamma^{m} \bar{\lambda}\right)^{\alpha}=0$, and $w_{\alpha}$ and $\widehat{\bar{w}}^{\alpha}$ are defined to have no poles with each other or with $\left(\bar{\Gamma}_{m}, \Gamma^{m}\right)$. In terms of $w_{\alpha}$ and $\hat{\bar{w}}^{\alpha}$, one finds that

$$
\begin{aligned}
\Omega_{\alpha}= & \frac{1}{4(\lambda \bar{\lambda})}\left[\frac{1}{2}\left(\bar{\lambda} \gamma^{m n}\right)_{\alpha}\left(\lambda \gamma_{m n} w\right)+\bar{\lambda}_{\alpha}\left(-\lambda^{\beta} w_{\beta}+4 u_{m} v^{m}\right)\right] \\
& +\frac{1}{\widehat{\gamma}} \frac{(\lambda \bar{\lambda})\left(\bar{\lambda} \gamma_{m} \gamma_{n}\right)_{\alpha}+\bar{\lambda}_{\alpha}\left(\lambda \gamma_{m} \gamma_{n} \bar{\lambda}\right)}{4(\lambda \bar{\lambda})^{2}} \Gamma^{m} \Gamma^{n}+\left(\lambda \gamma^{m}\right)_{\alpha} v_{m}, \\
\bar{w}^{\alpha}= & \widehat{\bar{w}}^{\alpha}+\frac{1}{2(\lambda \bar{\lambda})}\left[-u^{m}\left(\gamma_{m} \Omega\right)^{\alpha}+2 v_{m} u^{m} \lambda^{\alpha}-\left(\lambda \gamma_{m} \gamma_{n}\right)^{\alpha}\left(\Gamma^{m} \bar{\Gamma}^{n}-\frac{1}{2} \widehat{\gamma}^{m} \bar{\Gamma}^{n}\right)\right] .
\end{aligned}
$$

The next step to relating (3.7) to the pure spinor BRST operator is to perform the similarity transformation $Q_{\mathrm{B}-\mathrm{RNS}-\mathrm{GSS}} \rightarrow e^{-R^{\prime}} Q_{\mathrm{B}-\mathrm{RNS}-\mathrm{GSS}} e^{R^{\prime}}$ where

$$
R^{\prime}=\int d z\left[-\widehat{\xi} e^{-2 \widehat{\phi}} \frac{\left(\bar{\lambda} \gamma_{m n p q r} \bar{\lambda}\right)}{120(\lambda \bar{\lambda})^{2}} \Gamma^{m} \Gamma^{n} \Gamma^{p} \Gamma^{q} \Gamma^{r}+\widehat{\xi} e^{-\widehat{\phi}} \frac{\left(\bar{\lambda} \gamma_{m n p} r\right)}{24(\lambda \bar{\lambda})^{2}} \Gamma^{m} \Gamma^{n} \Gamma^{p}\right]
$$

One finds that

$$
\begin{aligned}
& e^{-R^{\prime}} Q_{\text {B-RNS-GSS }} e^{R^{\prime}}= \\
& \int d z\left[\lambda^{\alpha} d_{\alpha}+\widetilde{\bar{w}}^{\alpha} r_{\alpha}+c T-b c \partial c-\widehat{\eta}+\Gamma^{m} \frac{\left(\bar{\lambda} \gamma_{m} \gamma_{n} \lambda\right)}{2(\lambda \bar{\lambda})} \Pi^{n}\right. \\
& \quad+\Gamma^{m} \Gamma^{n}\left[\frac{\left(\bar{\lambda} \gamma_{m n p} r\right)}{8(\lambda \bar{\lambda})^{2}} \Pi^{p}+\frac{\left(r \gamma_{m n p} r\right)}{8(\lambda \bar{\lambda})^{2}} \bar{\Gamma}^{p}\right]+\Gamma^{m} \Gamma^{n} \Gamma^{p}\left[\frac{\left(\bar{\lambda} \gamma_{m n p} \partial \bar{\lambda}\right)}{24(\lambda \bar{\lambda})^{2}}-(\bar{\lambda} \theta) \frac{\left(\bar{\lambda} \gamma_{m n p} r\right)}{12(\lambda \bar{\lambda})^{2}}\right] \\
& \quad+\widehat{\gamma}\left(b+\bar{\Gamma}^{m} \Pi_{m}+\frac{\bar{\Gamma}_{m} \bar{\Gamma}_{n}}{4(\lambda \bar{\lambda})}\left(\lambda \gamma^{m n} r\right)+s^{\alpha} \partial \bar{\lambda}_{\alpha}+\Omega_{\alpha} \partial \theta^{\alpha}\right) \\
& \left.\quad+u_{m}\left(\bar{\Gamma}^{m}+\frac{1}{2(\lambda \bar{\lambda})} \bar{\lambda} \gamma^{m} d-\frac{\left(\bar{\lambda} \gamma^{m} \gamma^{n p} r\right)}{8(\lambda \bar{\lambda})^{2}} N_{n p}\right)\right]
\end{aligned}
$$

where

$$
\widetilde{\bar{w}}^{\alpha} \equiv \widehat{\bar{w}}^{\alpha}+\frac{1}{2(\lambda \bar{\lambda})}\left(\lambda \gamma_{m} \gamma_{n}\right)^{\alpha}\left(u_{m} v_{n}-\Gamma_{m} \bar{\Gamma}_{n}\right), \quad N_{m n}=\frac{1}{2}\left(\lambda \gamma_{m n} w\right)
$$

Since $\widetilde{\bar{w}}^{\alpha}$ of (3.13) commutes with the constraints $v^{m}\left(\gamma_{m} \bar{\lambda}\right)^{\alpha}=\bar{\Gamma}^{m}\left(\gamma_{m} \bar{\lambda}\right)^{\alpha}=0$ up to the gauge transformation $\delta \widetilde{\bar{w}}^{\alpha}=f^{m}\left(\bar{\lambda} \gamma_{m}\right)^{\alpha}$, one can easily verify that (3.12) also commutes with these constraints.

The BRST operator of (3.12) is constructed out of the operators discussed in [7] where the last line of (3.12) is $u_{m}$ times the constraint in [7] for $\bar{\Gamma}^{m}$ and the second-to-last line of (3.12) is $\widehat{\gamma}$ times the composite $b$ ghost expressed in terms of $\bar{\Gamma}^{m}$. After applying the similarity transformation $\mathcal{O} \rightarrow e^{-R^{\prime \prime \prime}} e^{-R^{\prime \prime}} \mathcal{O} e^{R^{\prime \prime}} e^{R^{\prime \prime \prime}}$ where

$$
\begin{aligned}
R^{\prime \prime} & =\int d z \frac{1}{2(\lambda \bar{\lambda})} \Gamma^{m}\left[-\bar{\lambda} \gamma^{m} d+\frac{\left(\bar{\lambda} \gamma^{m} \gamma^{n p} r\right)}{4(\lambda \bar{\lambda})} N_{n p}\right], \\
R^{\prime \prime \prime} & =\int d z \widehat{\gamma} v^{m}\left(\Pi_{m}+\frac{\left(\lambda \gamma^{m} \gamma^{n} r\right)}{4(\lambda \bar{\lambda})} \bar{\Gamma}_{n}\right),
\end{aligned}
$$


(3.12) reduces to

$$
\begin{aligned}
& e^{-R^{\prime \prime \prime}} e^{-R^{\prime \prime}} e^{-R^{\prime}} Q_{\text {B-RNS-GSS }} e^{R^{\prime}} e^{R^{\prime \prime}} e^{R^{\prime \prime \prime}}= \\
& \quad \int d z\left(\lambda^{\alpha} d_{\alpha}+\widetilde{\widetilde{w}}^{\alpha} r_{\alpha}-\widehat{\eta}+\widehat{\gamma}\left[b-B+v_{m}\left(\lambda \gamma^{m}\right)_{\alpha} \partial\left(\frac{\Gamma_{n}\left(\gamma^{n} \bar{\lambda}\right)^{\alpha}}{2(\lambda \bar{\lambda})}\right)\right]+u_{m} \bar{\Gamma}^{m}+c T-b c \partial c\right)
\end{aligned}
$$

where $B$ is the usual composite pure spinor $b$ ghost (ignoring normal ordering terms)

$$
\begin{aligned}
B= & -s^{\alpha} \partial \bar{\lambda}_{\alpha}+\frac{\bar{\lambda}_{\alpha}\left(2 \Pi^{m}\left(\gamma_{m} d\right)^{\alpha}-N_{m n}\left(\gamma^{m n} \partial \theta\right)^{\alpha}+\lambda^{\beta} w_{\beta} \partial \theta^{\alpha}\right)}{4(\bar{\lambda} \lambda)} \\
& -\frac{\left(\bar{\lambda} \gamma^{m n p} r\right)\left(d \gamma_{m n p} d+24 N_{m n} \Pi_{p}\right)}{192(\bar{\lambda} \lambda)^{2}}+\frac{\left(r \gamma_{m n p} r\right)\left(\bar{\lambda} \gamma^{m} d\right) N^{n p}}{16(\bar{\lambda} \lambda)^{3}}-\frac{\left(r \gamma_{m n p} r\right)\left(\bar{\lambda} \gamma^{p q r} r\right) N^{m n} N_{q r}}{128(\bar{\lambda} \lambda)^{4}} .
\end{aligned}
$$

Finally, the similarity transformation $\mathcal{O} \rightarrow e^{-U} \mathcal{O} e^{U}$ where

$$
U=\int d z c\left(B-v_{m}\left(\lambda \gamma^{m}\right)_{\alpha} \partial\left(\frac{\Gamma_{n}\left(\gamma^{n} \bar{\lambda}\right)^{\alpha}}{2(\lambda \bar{\lambda})}\right)-\widehat{\beta} \partial c\right)
$$

transforms (3.15) into

$$
e^{-U} e^{-R^{\prime \prime \prime}} e^{-R^{\prime \prime}} e^{-R^{\prime}} Q_{\text {B-RNS-GSS }} e^{R^{\prime}} e^{R^{\prime \prime}} e^{R^{\prime \prime \prime}} e^{U}=\int d z\left(\lambda^{\alpha} d_{\alpha}+\widetilde{\bar{w}}^{\alpha} r_{\alpha}+\widehat{\gamma} b+u_{m} \bar{\Gamma}^{m}\right)-\widehat{\eta}_{0} .
$$

The usual quartet argument implies that the cohomology of (3.18) is independent of

$$
\left(u_{m}, v^{m} ; \Gamma_{m}, \bar{\Gamma}^{m} ; \widehat{\gamma}, \widehat{\beta} ; b, c ; \bar{\lambda}_{\alpha}, \widetilde{\bar{w}}^{\alpha} ; r_{\alpha}, s^{\alpha}\right)
$$

so one recovers the original pure spinor BRST operator $Q_{\text {pure }}=\int d z \lambda^{\alpha} d_{\alpha}$ in the small Hilbert space defined with $\widehat{\eta}_{0}$. Note that this last similarity transformation with $U$ of (3.17) shifts the Virasoro $b$ ghost to

$$
e^{-U} b e^{U}=b+B-v_{m}\left(\lambda \gamma^{m}\right)_{\alpha} \partial\left(\frac{\Gamma_{n}\left(\gamma^{n} \bar{\lambda}\right)^{\alpha}}{2(\lambda \bar{\lambda})}\right)-\widehat{\beta} \partial c-\partial(\widehat{\beta} c) .
$$

So after twisting the spin-half $\psi^{m}$ variables into spin-zero and spin-one variables and performing a similarity transformation, the B-RNS-GSS BRST operator has been mapped into the pure spinor BRST operator. Finally, after gauging away the dependence on the non-minimal variables in (3.19), the unintegrated and integrated vertex operators of (2.16) and (2.17) are mapped into the usual pure spinor massless vertex operators

$$
V=\lambda^{\alpha} A_{\alpha}(x, \theta), \quad U=\int d z\left[\partial \theta^{\alpha} A_{\alpha}(x, \theta)+\Pi^{m} A_{m}(x, \theta)+d_{\alpha} W^{\alpha}(x, \theta)+\frac{1}{2} N^{m n} F_{m n}(x, \theta)\right] .
$$

\section{Acknowledgments}

I would like to thank CNPq grant 311434/2020-7 and FAPESP grants 2016/01343-7, 2019/24277-8 and 2019/21281-4 for partial financial support. 
Open Access. This article is distributed under the terms of the Creative Commons Attribution License (CC-BY 4.0), which permits any use, distribution and reproduction in any medium, provided the original author(s) and source are credited.

\section{References}

[1] D. Friedan, E.J. Martinec and S.H. Shenker, Conformal Invariance, Supersymmetry and String Theory, Nucl. Phys. B 271 (1986) 93 [INSPIRE].

[2] N. Berkovits, Super Poincaré covariant quantization of the superstring, JHEP 04 (2000) 018 [hep-th/0001035] [INSPIRE].

[3] W. Siegel, Classical Superstring Mechanics, Nucl. Phys. B 263 (1986) 93 [inSPIRE].

[4] N. Berkovits, Relating the RNS and pure spinor formalisms for the superstring, JHEP 08 (2001) 026 [hep-th/0104247] [INSPIRE].

[5] M. Kroyter, Superstring field theory in the democratic picture, Adv. Theor. Math. Phys. 15 (2011) 741 [arXiv:0911.2962] [INSPIRE].

[6] N. Berkovits, Covariant Map Between Ramond-Neveu-Schwarz and Pure Spinor Formalisms for the Superstring, JHEP 04 (2014) 024 [arXiv:1312.0845] [INSPIRE].

[7] N. Berkovits, Dynamical twisting and the b ghost in the pure spinor formalism, JHEP 06 (2013) 091 [arXiv: 1305. 0693] [INSPIRE]. 